\title{
RESISTÊNCIA À COLISTINA EM ISOLADOS DE HEMOCULTURAS DE RECÉM-NASCIDOS ADMITIDOS EM UM HOSPITAL ESCOLA
}

\author{
RESISTANCE TO COLISTIN IN ISOLATES OF NEWBORN \\ HEMOCULTURES ADMITTED IN A SCHOOL HOSPITAL
}

Roberta Filipini Rampelotto', Flávia Padilha de Freitas², Silvana Silveira Coelho²

Vinicius Victor Lorenzoni ${ }^{3}$, Marissa Bolson Serafin ${ }^{3}$, Rosmari Horner ${ }^{4}$.

\section{RESUMO}

O objetivo deste estudo foi avaliar a resistência frente à colistina em isolados de hemoculturas de recém-nascidos (RN) admitidos em um Hospital Escola no período de 4 anos. Os testes de identificação e os perfis de sensibilidade frente aos antimicrobianos foram realizados através de sistemas automatizados. No período deste estudo, 923 hemoculturas foram positivas, 92,09\% (850/923) causadas por microrganismos Gram-positivos e 7,91\% (73/923) por Gram-negativos. Destas, dois isolados foram resistentes à colistina: Acinetobacter baumannii e Sphingomonas paucimobilis; e um isolado de Pseudomonas aeruginosa mostrou perfil intermediário a este antimicrobiano, apresentando também significativa resistência aos carbapenêmicos. Este perfil é preocupante uma vez que estes antimicrobianos constituem a última alternativa para o tratamento de infecções causadas por bacilos Gram-negativos multirresistentes. Os resultados deste estudo sinalizam para o seguimento do monitoramento desta resistência.

Descritores: Sepse. Recém-nascido. Colistina.

\begin{abstract}
The objective of this study was to evaluate resistance against colistin in isolates of blood cultures from newborns (NB) admitted in a School Hospital in a 4-year period. The identification tests and antimicrobial susceptibility profiles were performed using automated systems. At the period of this study, 923 blood cultures were positive, $92.09 \%$ (850/923) were caused by Gram-positive microorganisms and $7.91 \%$ (73/923) by Gram-negative. Of these, two isolates were resistant to colistin: Acinetobacter baumannii and Sphingomonas paucimobilis; and a Pseudomonas aeruginosa isolate showed an intermediate profile to this antimicrobial, also presenting resistance to carbapenems. This profile is worrying since these antimicrobials are the last alternative for the treatment of infections caused by multiresistant Gram-negative bacilli. The results of this study indicate the follow-up of the monitoring of this resistance.
\end{abstract}

${ }^{1}$ Doutoranda do Programa de Pós-Graduação em Ciências Farmacêuticas (PPGCF) da Universidade Federal de Santa Maria (UFSM), Santa Maria, Rio Grande do Sul, Brasil.

${ }^{2}$ Aluna do Curso de Farmácia, Universidade Federal de Santa Maria (UFSM), Santa Maria, Rio Grande do Sul, Brasil.

${ }^{3}$ Mestrando(a) do Programa de Pós-Graduação em Ciências Farmacêuticas, Universidade Federal de Santa Maria (UFSM), Santa Maria, Rio Grande do Sul, Brasil.

${ }^{4}$ Professora do Departamento de Análises Clínicas e Toxicológicas, da Universidade Federal de Santa Maria (UFSM), Santa Maria, Rio Grande do Sul, Brasil. 


\section{Introdução}

Infecções hospitalares representam um sério problema de saúde pública, em razão de sua frequência morbimortalidade e custos de tratamento ${ }^{1,2}$. No período neonatal, as Infecções de Corrente Sanguínea (ICS) representam uma das complicações infecciosas mais recorrentes e perigosas consequentes de hospitalização, prolongando o tempo uma das complicações infecciosas mais recortalização dos recém-nascidos (RN) ${ }^{1,3-5}$.

As ICS causadas por microrganismos Gram-negativos multirresistentes (MDR) têm sido reconhecidas em todo mundo e constituem um dos maiores problemas nas Unidades de Terapia Intensiva Neonatal (UTI-RN), devido às elevadas taxas de morbidade e mortalidade ${ }^{6-10}$. 0 uso extensivo dos carbapenêmicos no tratamento das infeccõo causadas por estes microrganismos contribuiu para o desenvolvimento de mecanismos de resistência, limitando as opções terapêuticas, fazendo que houvesse o retorno da antibioticoterapia as polimixinas ${ }^{6,11-17}$.

As polimixinas foram introduzidas na terapêutica na década de 50, porém devido sua nefrotoxicidade foram substituídas por outros fármacos ${ }^{6,18,19}$. Estas, foram reintroduzidas na prática clínica na década de 90 , sendo que a polimixina $B$ e a polimixina $E$ (colistina), associadas a outros antimicrobianos, como os aminoglicosídeos e a tigeciclina constituem o tratamento de última escolha na terapia clínica para isolados resistentes aos carbapenêmicos ${ }^{6,18,19}$.

Devido ao longo tempo em que as polimixinas ficaram em desuso, as taxas de sensibilidade a este antimicrobiano ainda são elevadas ${ }^{20}$. Porém, alguns estudos têm demonstrado a rápida disseminação de isolados resistentes a colistina em pacientes adultos, principalmente na América Latina e Ásia, ${ }^{11-14,16}$ sendo raras as pesquisas que reportam sobre o seu perfil de sensibilidade em isolamentos de pacientes neonatais, bem como seu uso6. Ainda, em uma pesquisa recente, amostras resistentes a este antimicrobiano foram relatadas em isolados clínicos provenientes de hemoculturas de pacientes adultos admitidos em um centro de cuidados terciários na Hungria ${ }^{21}$

O objetivo deste estudo foi avaliar a resistência bacteriana frente à colistina em RN admitidos em um Hospital Escola no Rio Grande do Sul (RS) no período de 4 anos (junho de 2012-junho de 2016).

\section{Metodologia}

Foi realizado um estudo retrospectivo das hemoculturas positivas dos pacientes recém-nascidos admitidos em um Hospital Escola no Rio Grande do Sul por um periodo de quatro anos, de junho de 2012 a junho de 2016.

As culturas de sangue foram solicitadas quando havia presença de algum sinal clínico, como: aumento da temperatura $\left(>38^{\circ} \mathrm{C}\right)$ ou hipotermia $\left(<36^{\circ} \mathrm{C}\right)$, leucocitose $\left(>10.000\right.$ leucócitos $/ \mathrm{mm}^{3}$, especialmente com desvio à esquerda) ou granulocitopenia absoluta $\left(<1000\right.$ leucócitos $\left./ \mathrm{mm}^{3}\right)$.

As hemoculturas coletadas foram analisadas através do sistema automatizado BACTEC 9240® (Becton Dickinson, Sparks, MD). As culturas de sangue foram consideradas positivas quando houve 0 crescimento de um ou mais microrganismos em cada amostra. Na sequência foi realizada a bacterioscopia direta do caldo de cultura (coloração pelo método de Gram) e a semeadura em placas contendo ágar chocolate, ágar MacConkey e ágar sangue azida.

Após a semeadura, as placas foram incubadas em estufa bacteriológica a $35^{\circ} \mathrm{C} \pm 2^{\circ} \mathrm{C}$, por 18 a 24 horas em microaerofilia (ágar chocolate e ágar sangue azida). Os testes de identificação e os perfis de sensibilidade frente aos antimicrobianos das bactérias isoladas nos anos de 2012 e 2013 foram efetuados utilizando o sistema automatizado MicroScanß (Siemens); já os isolados coletados no período de 2014, 2015 e 2016 tiveram a identificação e os perfis de sensibilidade realizados através do aparelho automatizado VITEK®2-bioMérieux (France). Os mesmos foram interpretados em sensível, intermediário ou resistente de acordo com Clinical and Laboratory Institute (CLSI) vigente de cada ano de estudo ${ }^{22-26}$

O presente estudo foi aprovado pelo Comitê de Ética em Pesquisa da Universidade Federal de Santa Maria, sob o número 38850614.4 .0000 .5346$.

\section{Resultados e Discussão}

No período de estudo 923 hemoculturas foram positivas, sendo 92,09\% (850/923) causadas por microrganismos Gram-positivos e 7.91\% (73/923) por bactérias Gram-negativas. Destes isolados, dois apresentaram resistência frente à colistina: A. baumannii isolado no ano de 2014, com concentração inibitória mínima (CIM) $=4 \mu \mathrm{g} / \mathrm{mL}$; e Sphingomonas paucimobilis ( $\mathrm{CIM} \geq 16 \mu \mathrm{g} / \mathrm{mL}$ ) isolada no ano de 2015. Neste mesmo ano (2015) um isolado de $P$. aeruginosa (CIM $=4$ $\mu \mathrm{g} / \mathrm{mL}$ ) apresentou perfil intermediário frente a este antimicrobiano.

Em relação às unidades de internação dos $\mathrm{RN}$, cada paciente estava admitido em uma unidade diferente. 0 paciente que foi detectado $A$. baumannii encontrava-se internado na UTI-RN. Já os RN em que foram isolados $P$. aeruginosa e $S$. paucimobilis estavam admitidos no ambulatório e no setor de ginecologia e obstetrícia, respectivamente.

0 perfil de sensibilidade dos três isolados com perfil intermediário ou resistente à colistina, encontra-se descrito na Tabela 1

Tabela 1 - Perfil de sensibilidade dos três isolados com perfil intermediário ou resistente a colistina isolados de hemoculturas de RN.

\begin{tabular}{|c|c|c|c|c|c|c||c|c|c|}
\hline \multirow{2}{*}{ Antimicrobiano } & \multicolumn{7}{|c|}{ Microrganismo } & \multicolumn{3}{|c|}{ P. aeruginosa } & \multicolumn{2}{|c|}{ S. paucimobilis } & \\
\cline { 2 - 11 } & \multicolumn{3}{|c|}{ A. baumannii } & \multicolumn{1}{c|}{} \\
\hline & $\mathrm{S}$ & $\mathrm{R}$ & $\mathrm{NT}$ & $\mathrm{S}$ & $\mathrm{R}$ & $\mathrm{NT}$ & $\mathrm{S}$ & $\mathrm{R}$ & $\mathrm{NT}$ \\
\hline Amicacina & $\mathrm{x}$ & & & $\mathrm{x}$ & & & $\mathrm{x}$ & & \\
\hline Ampicilina & & $\mathrm{x}$ & & & $\mathrm{x}$ & & & & $\mathrm{x}$ \\
\hline $\begin{array}{c}\text { Ampicilina/ } \\
\text { sulbactam }\end{array}$ & $\mathrm{x}$ & & & & $\mathrm{x}$ & & & & $\mathrm{x}$ \\
\hline Cefepime & $\mathrm{x}$ & & & $\mathrm{x}$ & & & $\mathrm{x}$ & & \\
\hline Ceftriaxona & & $\mathrm{x}$ & & & & $\mathrm{x}$ & $\mathrm{x}$ & & \\
\hline Ceftazidima & $\mathrm{x}$ & & & & $\mathrm{x}$ & & $\mathrm{x}$ & & \\
\hline Ciprofloxacino & $\mathrm{x}$ & & & $\mathrm{x}$ & & & $\mathrm{x}$ & & \\
\hline Gentamicina & $\mathrm{x}$ & & & $\mathrm{x}$ & & & $\mathrm{x}$ & & \\
\hline Imipenem & $\mathrm{x}$ & & & & $\mathrm{x}$ & & $\mathrm{x}$ & & \\
\hline Meropenem & $\mathrm{x}$ & & & & $\mathrm{x}$ & & $\mathrm{x}$ & & \\
\hline $\begin{array}{c}\text { Piperacilina/ } \\
\text { Tazobactam }\end{array}$ & & & $\mathrm{x}$ & & $\mathrm{x}$ & & $\mathrm{x}$ & & \\
\hline Tigeciclina & $\mathrm{x}$ & & & & $\mathrm{x}$ & & & & $\mathrm{x}$ \\
\hline Polimixina B & & & $\mathrm{x}$ & & $\mathrm{x}$ & & & & $\mathrm{x}$ \\
\hline Tobramicina & & & $\mathrm{x}$ & & & $\mathrm{x}$ & & $\mathrm{x}$ & \\
\hline
\end{tabular}

$\mathrm{S}=$ sensível; $\mathrm{R}=$ resistente; $\mathrm{NT}=$ não testado

Nesta pesquisa 923 hemoculturas foram positivas, 92,09\% bactérias Gram-positivas e 7,91\% microrganismo Gram-negativos. Um estudo realizado por Viana et al. (2011) $)^{27}$, avaliando hemoculturas de RN em um hospital da rede privada em Campina Grande (Paraíba), demonstrou o predomínio das bactérias Gram-positivas, 86,67\%, corroborando com nossa pesquisa. Já na pesquisa realizada por Oliveira et al. $(2012)^{5}$, avaliando o perfil etiológico de hemoculturas de pacientes internados em UTI-RN no estado de Mato Grosso, houve o predomínio de bactérias Gram-negativas $(50 \%)$ seguidos das Gram-positivas $(29,3 \%$ e $20,7 \%$ de fungos. Essa diferença encontrada pode ser explicada, pelo fato de que estes índices podem variar entre os hospitais, de acordo com a região geográfica ${ }^{28}$.

Entre os microrganismos isolados, A. baumannii, e S. paucimobilis apresentaram resistência à colistina; e $P$. 
aeruginosa um perfil intermediário frente a este antimocrobiano. Bactérias Gram-negativas não fermentadoras, como $P$. aeruginosa, $A$. baumannii e $S$. paucimobilis estão amplamente disseminadas no ambiente e estão cada vez mais associadas a infecções nosocomiais graves ${ }^{29}$. S. paucimobilis é considerado um patógeno oportunista, sendo raramente isolado em seres humanos ${ }^{30}$. Embora as infecções associadas a este microrganismo sejam consideradas de meno significância clínica, já têm sido relatada sua presença em contextos clínicos, como bacteremia/septicemia causada por soluções contaminadas, como por exemplo água destilada ${ }^{31,32}$

Um estudo prospectivo realizado no mesmo nosocômio deste estudo, no período de seis meses (agosto de 2015 a janeiro de 2016), avaliando isolados de bacilos Gram-negativos não fermentadores, de diversos espécimes clínicos, de todos os pacientes admitidos neste hospital, encontraram um total de 293 isolados, sendo este tipo de resistência apresentado em $9(3,89 \%)$ cepas de $P$. aeruginosa ${ }^{16}$. Isolados de A. baumannii também já foram reportados com este tipo de resistência, e até mesmo cepas panresistentes, conforme foi demonstrado em um estudo avaliando isolados clínicos e culturas de vigilância epidemiológica obtidas de pacientes admitidos na Unidade de Terapia Intensiva adulta (UTI) de um hospital terciário na Espanha, no qual pode-se observar a rápida habilidade que estes microrganismos tem em adquirir resistência ${ }^{33}$.

Uma pesquisa conduzida na Índia no período de cinco meses analisando isolados de $P$. aeruginosa e $A$ baumannii obtidos de diversos espécimes clínicos como sanque, pus, urina e secreções respiratórias inferiores (lavado broncoalveolar, lavado brônquico e secreção endotraqueal) de pacientes hospitalizados, relatou $8 \%$ de resistência à colistina ${ }^{34}$. No Irã, um estudo realizado por Bahador et al. $(2013)^{35} \mathrm{em}$ Unidades de Terapia Intensiva terciárias de três hospitais universitários do norte, centro e sul do país, selecionou 91 isolados de A. baumannii de diversos espécimes clínicos, dos quais $14,2 \%$ foram resistentes à colistina e $9 \%$ dos isolados apresentaram resistência simultânea à colistina, rifampicina e tigeciclina, sendo que todos os isolados resistentes à colistina também foram resistentes à polimixina $\mathrm{B}$.

Juhász et al. (2017) $)^{21}$ avaliaram isolados de hemoculturas no período compreendido entre 2010 e 2011 e também no ano de 2016 de pacientes admitidos em um centro de cuidados terciários em Budapeste, Hungria, e encontraram $0,6 \%, 1,3 \%$, e 2,6\% de resistência a colistina para família Enterobacteriaceae, Pseudomonas spp. e Acinetobacter spp., respectivamente. Ainda, o programa de vigilância de resistência Antimicrobial Surveillance Program (SENTRY) durante o período 2006 a 2009 avaliou isolados clínicos de diversos sítios de infecção, coletados em várias partes do mundo, no qual foi observado resistência à colistina em $P$. aeruginosa de $0,6 \%$ na região Ásia-Pacífico, $0,4 \%$ na Europa, $0,3 \%$ na América Latina e $0,1 \%$ na América do Norte. Já para $A$. baumannii a resistência foi de $0,9 \%$ na Europa, 1,1\% América Latina e 2,0\% na América do Norte ${ }^{36}$.

Em relação ao outro microrganismo isolado neste estudo, S. paucimobilis, é raramente encontrado em infecções em seres humanos, o qual geralmente é encontrado como um contaminante hospitalar, sendo assim, medidas de controle e prevenção de infecção hospitalar necessitam ser intensificadas, visando prevenir a transmissão cruzada e surtos hospitalares ${ }^{30}$. Krishna et al. (2011) ${ }^{30}$, relataram um caso de S. paucimobilis isolado de uma infecção do trato urinário em um receptor de transplante renal, avaliando a resistência do isolado a toda a primeira, segunda e terceira linha de antibióticos testados para bacilos Gram-negativos de rotina, incluindo colistina, apresentando sensibilidade apenas à vancomicina e à rifampicina

A resistência a colistina constitui um sério problema, já que em 2013, a Agência Nacional de Vigilância Sanitária (ANVISA) publicou uma Nota Técnica que contém medidas de prevenção e controle de infecções por enterobactérias multirresistentes, a qual preconiza que o tratamento das bactérias multirresistentes aos carbapenêmicos seja realizado com politerapia antimicrobiana, e sugere a utilização de polimixina ( $\mathrm{B}$ ou E), associada com um ou mais antimicrobianos (aminoglicosídeos), baseado nos perfis locais ${ }^{37}$. Ainda, a ampla utilização das polimixinas na veterinária para o tratamento de infecções gastrointestinais causadas por bactérias da família Enterobacteriaceae, bem como no ambiente hospitalar tem contribuído para mutações mediadas pelo gene mcr-1 para codificar a resistência à colistina em bacilos Gramnegativos, aumentando os índices desta resistência ${ }^{15,38,39}$

Em nosso estudo foram avaliados apenas isolados de hemoculturas de RN. Santos et al. $(2016)^{16}$ encontraram entre os espécimes em que predominou o isolamento de $P$. aeruginosa resistente à colistina, secreções de tecidos moles $(33,33 \%)$, seguido de secreções do trato respiratório $(22,22 \%)$. Ainda, relataram que a idade prevalente dos pacientes fo entre 41 a 69 anos $(77,77 \%)^{16}$. Bahador et al. (2013)35 nos isolados com resistência simultânea à colistina, rifampicina e tigeciclina, demonstraram que a prevalência foi nos espécimes do trato respiratório $(75 \% ; 6 / 8)$, seguido de um isolado de ferida operatória e outro de um paciente com infecção do trato urinário.

Em relação aos setores de internação, cada RN estava em uma unidade: Ambulatório, Ginecologia e Obstetrícia e UTI-RN. Santos et al. $(2016)^{16}$ relataram que os isolados de $P$. aeruginosa e A. baumannii resistentes a colistina tiveram maior frequência na Unidade de Terapia Intensiva, $42,85 \%$, seguido do pronto atendimento $(23,80 \%)$.

Quanto ao perfil de sensibilidade dos microrganismos, os três isolados resistentes a colistina apresentaram $100 \%$ de sensibilidade frente à amicacina, cefepime, ciprofloxacino e gentamicina apresentaram; e 66,67\% de sensibilidade frente ao imipenem e meropenem. Santos et al. $(2016)^{16}$ demonstraram $78 \%$ de sensibilidade frente à amicacina e $56 \%$ à gentamicina nos isolados de $P$. aeruginosa, diferentemente do nosso estudo.

Ainda, nossa pesquisa mostrou que os isolados de $A$. baumannii e $P$. aeruginosa $(100 \%-2 / 2)$ foram resistentes a ampicilina. Santos et al. $(2016)^{16}$ verificaram $100 \%$ de resistência a ampicilina/sulbactam e $67 \%$ dos isolados foram resistentes a ceftazidima, imipenem e meropenem. Bahador et al. $(2013)^{35}$ demonstraram que todos os isolados foram resistentes ao ceftriaxona e cefepime, e relataram $4,9 \%$ de isolados resistentes ao imipenem e à colistina simultaneamente porém todos sensíveis à tigeciclina. Ainda estes pesquisadores verificaram resistência a rifampicina em $77 \%$ dos isolados ${ }^{35}$. Uma pesquisa realizada com 60 isolados de A. baumannii coletados de pacientes e do ambiente em um hospital do Rio de Janeiro, apresentaram resistência igual ou superior a $80 \%$ para piperacilina/tazobactam, cefotaxima, ciprofloxacino, cefepime, imipenem e meropenem; e $68 \%$ de resistência para polimixina B, sendo que a resistência a colistina não foi relatada

Em 2016, aANVISA publicou um comunicado de risco sobre a detecção do gene mcr-1, responsável pela resistência à polimixina mediada por plasmídeos, que traz as medidas a serem adotadas pelos laboratórios de microbiologia, no qual as Comissões de Controle de Infecção Hospitalar $(\mathrm{CClH})$ devem ser informadas quando forem isolados Escherichia coli com CIM $\geq 4 \mathrm{mg} / \mathrm{L}$ para polimixina. ${ }^{40}$ Estes isolados, quando detectados, devem ser notificados a ANVISA e após encaminhados ao Laboratório Central de Saúde Pública (LACEN) para realização de testes de confirmação, além da adoção de medidas de controle para o uso das polimixinas. ${ }^{40}$ No Hospital Escola em questão, sabe-se que ações ativas da $\mathrm{CCIH}$, envolvendo a capacitação dos profissionais que atuam neste nosocômio, além da conduta rigorosa na dispensação dos antimicrobianos, contribui para a prevenção e o controle da disseminação e propagação de isolados MDR.

Considerações Finais

Dois isolados apresentaram resistência a colistina, e outro ( $P$. aeruginosa) mostrou perfil intermediário frente a este antimicrobiano, mas foi resistente aos carbapenêmicos, demonstrando preocupação, já que estes antimicrobianos constituem a última alternativa para o tratamento de infecções causadas por bacilos Gram-negativos MDR, sendo que estes isolados resistentes foram provenientes da corrente sanguínea de RN, os quais são mais vulneráveis à infecções e estão iniciando o seu sistema de defesa. Assim, o monitoramento desta resistência deve ser realizado, os resultados relatados as autoridades sanitárias como a ANVISA, e medidas rigorosas pela comissão de Infecções Relacionadas à Assistência à Saúde (IRAS) devem ser adotadas.

Referências

1. Sousa MA, Medeiros NM, Cardoso AM, Carneiro JR. Microrganismos prevalentes em hemoculturas de pacientes da Unidade de Terapia Intensiva de um Hospital Escola de Goiânia-GO, entre 2010 e 2013. Estudos. 2014; 41(3). 627-635. 2. World Health Organization. The evolving threat of antimicrobial resistance: Options for action. WHO Publ. 2012;1-119. 3. Agarwal A, Bhat S. Clinico-microbiological study of neonatal sepsis. J Intern Med Dent. 2015; 2(1): $22-29$. 4. Fjalstad JW, Stensvold HJ, Bergseng H, Simonsen GS, Salvesen B, Ronnestad AE, et al. Early-onset sepsis and antibiotic exposure in term infants: A Nationwide population-based study in Norway. Ped Infect Dis J. 2016; 35(1): 1-6.

5. Oliveira MLA, Veronesi CL, Goulart LS. Caracterização de recém-nascidos com hemoculturas positivas internados em Unidade de Terapia Intensiva Neonatal. Gestão \& Saúde. 2012; 3(2): 438-450.

6. Al-lawama M, Aljbour H, Tanash A, Badran E. Intravenous Colistin in the treatment of multidrugresistant Acinetobacter in neonates. Ann Clin Microbiol Antimicrob. 2016; 15: 8. 
7. Patel SJ, Saiman L. Antibiotic resistance in NICU pathogens: Mechanisms, clinical impact, and prevention including antibiotic stewardship. Clinics in Perinatology. 2010; 37(3): 547-563.

8. Tzialla C, Borghesi A, Serra G, Stronati M, Corsello G. Antimicrobial therapy in neonatal intensive care unit. Ital J Ped. 2015; 41(1): 1-6.

9. Oliveira AC, Silva RS. Desafios do cuidar em saúde frente à resistência bacteriana: uma revisão. REE. 2008; 10(1): 189-197.

10. Paiano M, Bedendo J. Resistência antimicrobiana de amostras de Staphylococcus aureus isoladas de recém-nascidos saudáveis. REE. 2009; 11(4): 841-846.

11. Aires CAM, Pereira PS, Asensi MD, Carvalho-Assef APDA. mgrB mutations mediating polymyxin B resistance in Klebsiella pneumoniae isolates from rectal surveillance swabs in Brazil. Antimic Ag Chem. 2016; 60(11): 6969-6972.

12. Bassetti M, De Waele JJ, Eggimann P, Garnacho-Montero J, Kahlmeter G, Menichetti F, et al. Preventive and therapeutic strategies in critically ill patients with highly resistant bacteria. Int Care Med. 2015; 41(5): 776-795.

13. Gomes DBC, Genteluci GL, Carvalho KR, Medeiros LM, Almeida VC, De Castro E de AR, et al. Acinetobacter baumannil multirresistentes: emergência de resistência à polimixina no Rio de Janeiro. Vigil Sanit Debate. 2016; 4(3): 28-34.

14. Gurjar M. Colistin for lung infection: an update. J Inten Care. 2015; 3(1): 1-12.

15. Monaco M, Giani T, Raffone M, Arena F, Garcia-Fernandez A, Pollini S, et al. Colistin resistance superimposed to endemic carbapenem-resistant Klebsiella pneumoniae: a rapidly evolving problem in Italy, November 2013 to April 2014 Eurosurveillance. 2014; 19(42): 20939

16. Santos $S$, Rocca S, Hörner R. Colistin resistance in non-fermenting Gram-negative bacilli in a university hospital. BJID. 2016; 20(6): 649-650.

17. Vieira PB, Picoli SU. Acinetobacter baumannii multirresistente: Aspectos clínicos e epidemiológicos. RBCS. 2015; 19(2): 151-156.

18. Ordooei Javan A, Shokouhi S, Sahraei Z. A review on colistin nephrotoxicity. Eu J Clin Pharmacol. 2015; 71(7): 801-810.

19. Kaye KS, Pogue JM, Tran TB, Nation RL, Li J. Agents of last resort: polymyxin resistance. Infect Dis Clin North America. 2016; 30(2): 391-414

20. Girardello R, Gales AC. Resistência às Polimixinas: velhos antibióticos, últimas opções terapêuticas. R Epidemiol Control Infec. 2012; 2(2): 66-69.

21. Juhász E, Iván $M$, Pintér $E$, Pongrácz J, Kristóf $K$. Colistin resistance among blood culture isolates at a tertiary care centre in Hungary. J Global Antimicrob Resist. 2017; S2213-7165 (17): 30147-9.

22. Clinical And Laboratory Standards Institute (CLSI).Performance standards for antimicrobial susceptibility testing Twenty-third informational supplement, document M100-S22. Wayne, PA, USA: CLSI, 2012

23. Clinical And Laboratory Standards Institute (CLSI).Performance standards for antimicrobial susceptibility testing; Twenty-third informational supplement, document M100-S23. Wayne, PA, USA: CLSI, 2013.

24. Clinical And Laboratory Standards Institute (CLSI).Performance standards for antimicrobial susceptibility testing; Twenty-third informational supplement, document M100-S24. Wayne, PA, USA: CLSI, 2014.

25. Clinical and Laboratory Standards Institute (CLSI). Performance Standards for antimicrobial susceptibility testing; Twenty-six informational supplement, document M100-S25. Wayne, PA, USA: CLSI 2015.

26. Clinical and Laboratory Standards Institute (CLSI). Performance Standards for antimicrobial susceptibility testing Twenty-six informational supplement, document M100-S26. Wayne, PA, USA: CLSI 2016.

27. Viana APP, Soares R dos S, Castro ARL de, Kluczynik CEN, Catão RMR. Incidência bacteriana em hemoculturas de recém-nascidos e perfil de suscetibilidade frente aos antimicrobianos. BioFar. 2011; 5(1): 102-110.

28. Gohel K, Jojera A, Soni S, Gang S, Sabnis R, Desai M. Bacteriological profile and drug resistance patterns of blood culture isolates in a tertiary care nephrourology teaching institute. BioMed Res Inter. 2014; 2014: 1-6.

29. Dias VC. Resistência aos carbapenêmicos e virulência de Acinetobacter baumannii e Pseudomonas aeruginosa isolados de um serviço de saúde terciário. Juiz de Fora, 2015. 118 p. Tese (Doutorado) - Universidade Federal de Juiz de Fora, Instituto de Ciências Biológicas. Programa de Pós-Graduação em Ciências Biológicas: Imunologia e Genética, 2015.
30. Krishna S, Ciraj AM, Bairy I, Shobha KL. Sphingomonas paucimobilis urinary tract infection in a renal transplant recipient: a rare case. Inter J Med Public Health. 2011;1(1): 47-49.

31. Bayram N, Devrim I, Apa H, Gülfidan G, Türkyilmaz HN, Günay I. Sphingomonas paucimobilis infections in children 24 case reports. MJHID. 2013; 5(1):1-5.

32. Ryan MP, Adley CC. Sphingomonas paucimobilis: a persistent Gram-negative nosocomial infectious organism. J Hospit Infec. 2010; 75(3): 153-157.

33. Valencia R, Arroyo LA, Conde M, Aldana JM, Torres MJ, Fernández-Cuenca F, et al. Nosocomial outbreak of infection with pandrug-resistant Acinetobacter baumannii in a tertiary care university hospital. Infect Control Hosp Epidemiol. 2009; 30: $257-263$

34. Shanthi M, Sekar U. Multi-drug resistant Pseudomonas aeruginosa and Acinetobacter baumannii infections among hospitalized patients: risk factors and outcomes. J Assoc Phys Ind. 2009; 57(636): 638-645

35. Bahador A, Taheri M, Pourakbari B, Hashemizadeh Z, Rostami H, Mansoori N, et al. Emergence of Rifampicin Tigecycline, and Colistin-Resistant Acinetobacter baumannii in Iran; Spreading of MDR Strains of Novel International Clone Variants. Microbial Drug Resistance. 2013; 19(5): 397-406.

36. Gales AC, Jones RN, Sader HS. Contemporary activity of colistin and polymyxin B against a worldwide collection of Gram-negative pathogens: results from the SENTRY Antimicrobial Surveillance Program (2006-09). J Antimicrobia Chem. 2011; 66(9): 2070-2074.

37. BRASIL. Agência Nacional de Vigilância Sanitária (ANVISA). Microbiologia Clínica para o Controle de Infecção Relacionada à Assistência à Saúde. Módulo 6: Detecção e identificação de bactérias de importância médica. 9 volumes, 2013. $150 \mathrm{p}$.

38. Fernandes MR, Moura Q, Sartori L, Silva KC, Cunha MPV, Esposito F et al. Silent dissemination of colistin-resistant Escherichia coli in South America could contribute to the global spread of the mcr-1gene. Eurosurveillance. 2016; 21(17): 30214

39. Lentz SA, de Lima-Morales D, Cuppertino VM, Nunes L de S, da Motta AS, Zavascki AP, et al. Letter to the editor: Escherichia coli harbouring mcr-1 gene isolated from poultry not exposed to polymyxins in Brazil. Eurosurveillance. 2016; 21(26): 30267

40. BRASIL. Agência Nacional de Vigilância Sanitária (ANVISA). Comunicado de Risco № 01/2016 - GVIMS/GGTES/ ANVISA. Detecção do gene responsável pela resistência à polimixina mediada por plasmídeos (mcr-1) no Brasil. 06 de outubro de 2016.

Roberta Filipini Rampelotto

Endereço para correspondência - Universidade Federal de Santa Maria (UFSM), Prédio 26, Sala 1201, Campus da UFSM CEP: 97015-900, Santa Maria, RS, Brasi

E-mail: robertarampelotto@gmail.com Lattes: http://lattes.cnpq.br/3542868869006265

Flávia Padilha de Freitas - flavia.pdf@hotmail.com Vinicius Victor Lorenzoni - Iorenzoni.vinicius@gmail.com Marissa Bolson Serafin - mbs_1304@hotmail.com Silvana Silveira Coelho - silvana.scoelho@hotmail.com Rosmari Hörner - rosmari.ufsm@gmail.com

Enviado em 06 de Agosto de 2017. Aceito em 18 de Agosto de 2017. 